\title{
CHARACTERIZATIONS OF THE CONTINUOUS IMAGES OF THE PSEUDO-ARC
}

\author{
BY
}

\author{
LAWRENCE FEARNLEY
}

1. Introduction. At the Summer Institute on Set Theoretic Topology, Madison, Wisconsin, 1955, the question "What characterizes all continuous images of the pseudo-arc $[1 ; 7 ; 10]$ ?'” was raised by R. H. Bing [3]. In particular it was asked whether or not there exists a characterization of the continuous images of the pseudo-arc analogous to the well-known Hahn-Mazurkiewicz characterization of the continuous images of the arc $[5 ; 8]$. The purpose of this paper is to investigate this question and to initiate an investigation of the properties of the class of continuous images of all chainable continua in general. A related question considered is whether or not every chainable continuum is a continuous image of the pseudo-arc.

In $\S 3$ of this paper two global characterizations of the continuous images of the pseudo-arc are established $\left({ }^{1}\right)$, and it is shown that if the definition of local connectedness is suitably reformulated, then these characterizations are analogous to that of Hahn and Mazurkiewicz for the arc. Furthermore, in $\$ 4$, these characterizations prove to be readily applicable to showing that the pseudo-arc can be mapped continuously onto any chainable continuum. A consequence of this latter result is that the class of continuous images of the pseudo-arc and the class of continuous images of all chainable continua are identical.

Next we present one of the most important results of this paper with respect to answering the question of whether or not there exists a characterization of the continuous images of the pseudo-arc analogous to that of Hahn and Mazurkiewicz for the arc. Specifically, in $\S 5$, we show that there does not exist any local topological property which characterizes all continuous images of the pseudo-arc. This simultaneously indicates that the original question of R. H. Bing has an essentially negative answer and that, in this respect, the characterizations of $\$ 3$ cannot be improved. Finally, in $\S 6$, a series of examples is given to show that the

Presented to the Society, January 29, 1960 under the title Continuous mappings of the pseudoarc; received by the editors January 17, 1963.

(1) The referee has pointed out that during the period of his examination of this paper an alternative development of one of these characterizations was given by A. Lelek, On weakly chainable continua, Fund. Math. 51 (1963), 271-283. However, since the preliminary theorems of the present development as well as this characterization are necessary to the completion, in $\S 5$, of the answer to the question of $\mathbf{R}$. H. Bing, this paper is presented essentially in its original form. 
major generalizations of the class of locally connected compact metric continua and the class of chainable continua fail to be contained in the class of continuous images of the pseudo-arc. In this regard, therefore, the results of $\S 4$ are also the best possible.

In a later paper to be presented by the author the characterizations of the continuous images of the pseudo-arc established in this paper will be used to develop properties of the class of continuous images of all chainable continua, including the results that this class is closed under the operations of finite connected union and countable topological product.

Continua with similar geometric characteristics to those described in $\S 6$ have been discussed by M. K. Fort, Jr. [4] and S. Waraszkiewicz [11]. While these papers and this present study have different primary objectives it is of interest to note, in their investigations of continua which fail to be continuous images of any members of rather broad classes of continua, that similar types of examples were developed. One of the resulrs of $\S 4$ "that chainable continua are continuous images of the pseudo-arc," was also recently developed by J. Mioduszewski [9]. However, since the methods of this paper are very different from those of [9] and the result in question is a direct result and natural complement of the more general results of this paper, it is included here for completeness.

All continua considered in this paper will be separable metric continua.

2. Preliminaries. The more standard terms used in this paper are defined in [12] or in the other appropriately indicated references. In addition we shall define a number of special terms to be used throughout this paper. In general these latter terms and notations were suggested by those used by Bing in [1] and [2].

Definition 1. A p-chain will be defined to be a finite sequence of sets each of which, except the last, intersects its successor in the sequence. The members of the sequence are called links and the notation $P=\left(p_{1}, p_{2}, \cdots, p_{n}\right)$ will be used to denote the $p$-chain $P$ whose links are $p_{1}, p_{2}, \cdots, p_{n}$.

Definition 2. If $P$ is a $p$-chain, then the $p$-chain consisting of the links of $P$ in reverse order will be said to be the conjugate of $P$ and will be denoted by $\bar{P}$. A $p$-chain $\boldsymbol{P}$ such that $\boldsymbol{P}$ and $\bar{P}$ consist of the same sets in the same order will be said to be a self-conjugate $p$-chain. However, if $P=\left(p_{1}, p_{2}, \cdots, p_{n}\right)$ is a selfconjugate $p$-chain, the conjugate $\bar{P}=\left(p_{n}, p_{n-1}, \cdots, p_{1}\right)$ of $P$ is formally distinct from $P$ and will be considered as a different $p$-chain.

Definition 3. The $p$-chain sum $P+Q$ of $p$-chains $P=\left(p_{1}, p_{2}, \cdots, p_{n}\right)$ and $Q=\left(q_{1}, q_{2}, \cdots, q_{m}\right)$ will be defined if and only if $p_{n}$ and $q_{1}$ intersect. We will then define $P+Q$ to be the sequence of sets $p_{1}, p_{2}, \cdots, p_{n}, q_{1}, q_{2}, \cdots, q_{m}$.

Definition 4. We shall define the sub-p-chain $P(h, k), 1 \leqq h \leqq k \leqq n$, of the $p$-chain $P=\left(p_{1}, p_{2}, \cdots, p_{n}\right)$ to be the sequence of links $p_{h}, p_{h+1}, \cdots, p_{k}$. The notation $P(k, h), 1 \leqq h \leqq k \leqq n$, will be used to denote the sub- $p$-chain of $\bar{P}$ consisting of the sequence of links $p_{k}, p_{k-1}, \cdots, p_{h}$. 
Definition 5. If $P=\left(p_{1}, p_{2}, \cdots, p_{n}\right)$ and $Q=\left(q_{1}, q_{2}, \cdots, q_{m}\right)$ are $p$-chains and each link $p_{i}$ of $P$ is a subset of a link $q_{x_{i}}$ of $Q$, then the sequence of ordered pairs of integers $\left(1, x_{1}\right),\left(2, x_{2}\right), \cdots,\left(n, x_{n}\right)$ will be said to be a pattern of $P$ in $Q$. If, in this pattern, $\left|x_{i}-x_{j}\right| \leqq 1$ whenever $|i-j| \leqq 1,1 \leqq i, j \leqq n$, then the pattern will be said to be an r-pattern of $P$ in $Q$.

Definition 6. A $p$-chain $P$ will be said to be a refinement of a $p$-chain $Q$ if there is an $r$-pattern of $P$ in $Q$.

A number of types of refinements will be distinguished:

Definition 7. If a $p$-chain $P=\left(p_{1}, p_{2}, \cdots, p_{n}\right)$ has an $r$-pattern of the form $\left(1, x_{1}=1\right),\left(2, x_{2}\right), \cdots,\left(n, x_{n}=m\right)$ in a $p$-chain $Q=\left(q_{1}, q_{2}, \cdots, q_{m}\right)$ then $P$ will be said to be a normal refinement of $Q$.

DEFINITION 8. If a $p$-chain $P=\left(p_{1}, p_{2}, \cdots, p_{n}\right)$ has an $r$-pattern $\left(1, x_{1}\right),\left(2, x_{2}\right), \cdots$, $\left(n, x_{n}\right)$ in a $p$-chain $Q=\left(q_{1}, q_{2}, \cdots, q_{m}\right)$ and for each $i, i=1,2, \cdots, n, p_{i}$ is the same set as $q_{x_{i}}$, and if each link of $Q$ corresponds to at least one link of $P$, then $P$ will be said to be a principal refinement of $Q$.

If there is an $r$-pattern of $P$ in $Q$ satisfying the requirements of both 7 and 8 then $P$ will be said to be a principal normal refinement of $Q$.

Definition 9. If a $p$-chain $P=\left(p_{1}, p_{2}, \cdots, p_{n}\right)$ has an $r$-pattern $\left(1, x_{1}\right),\left(2, x_{2}\right), \cdots$, $\left(n, x_{n}\right)$ in a $p$-chain $Q=\left(q_{1}, q_{2}, \cdots, q_{m}\right)$, and for each pair of links $p_{h}$ and $p_{k}$ of $P$ for which $\left|x_{h}-x_{k}\right|>2$ the sub- $p$-chain $P(h, k)$ of $P$, or $\bar{P}$, has links $p_{r}$ and $p_{s}$ such that $r<s$ if $h<k$ and $r>s$ if $h>k$, and such that $\left|x_{h}-x_{s}\right|=1$ and $\left|x_{k}-x_{r}\right|=1$, then $P$ will be said to be crooked in $Q$.

In any type of refinement of a $p$-chain $P$ in a $p$-chain $Q$, there may be several $r$-patterns of $P$ in $Q$ having the appropriate properties. However, in referring to a refinement whose existence has been hypothesized or otherwise established, we will assume that a particular $r$-pattern has been chosen and that this $r$-pattern will remain fixed throughout the given argument. Thus, in these circumstances, we will speak of "the" $r$-pattern of a $p$-chain $P$ in a $p$-chain $Q$.

The concept of $p$-chainability of a continuum is now introduced.

Definition 10. A continuum $H$ will be said to be $p$-chainable if there is a sequence of $p$-chains $P_{1}, P_{2}, P_{3}, \cdots$ such that for each positive integer $i$ :

(a) The union of the elements of $P_{i}$ is $H$.

(b) $P_{i+1}$ is a normal refinement of $P_{i}$.

(c) The diameter of each link of $P_{i}$ is less than $1 / i$.

(d) The closure of each link of $P_{i+1}$ is a subset of the link of $P_{i}$ to which it corresponds under the $r$-pattern of $P_{i+1}$ in $P_{i}$.

A sequence of $p$-chains having these properties with respect to a continuum $H$ will be said to be associated with $H$.

It will develop that the concepts of $p$-chain and $p$-chainability are of fundamental importance in this investigation of the continuous images of the pseudo-arc and in the related subsequent study mentioned in the Introduction. 
3. Characterizations of the continuous images of the pseudo-arc. We shall show that the property of $p$-chainability and a modified somewhat stronger form of this property are each characterizations of the continuous images of the pseudoarc. Furthermore it will be shown that if the property of local connectedness is suitably reformulated as a global property then these characterizations are analogous to that of Hahn and Mazurkiewicz for the arc. It will be established in $\$ 5$ that no local characterization of the continuous images of the pseudo-arc exists so that in this respect these analogies cannot be improved.

A number of preliminary results will be needed, some of which will be of interest in themselves.

THEOREM 3.1. Each of the relations, "refinement," "normal refinement", and "principal refinement," between ordered pairs of p-chains, is transitive.

Proof. Let $P=\left(p_{1}, p_{2}, \cdots, p_{n}\right), \quad Q=\left(q_{1}, q_{2}, \cdots, q_{m}\right)$ and $T=\left(t_{1}, t_{2}, \cdots, t_{k}\right)$. Suppose first that $P$ is a refinement of $Q$ and $Q$ is a refinement of $T$. Then there is an $r$-pattern $\left(1, x_{1}\right),\left(2, x_{2}\right), \cdots,\left(n, x_{n}\right)$ of $P$ in $Q$ and an $r$-pattern $\left(1, y_{1}\right)$, $\left(2, y_{2}\right), \cdots,\left(m, y_{m}\right)$ of $Q$ in $T$. It follows from the definition of pattern that for each integer $i, 1 \leqq i \leqq n$, the link $p_{i}$ of $P$ is a subset of the link of $T$ with subscript $y_{x_{i}}$. Hence, $\left(1, y_{x_{1}}\right),\left(2, y_{x_{2}}\right), \cdots,\left(n, y_{x_{n}}\right)$ is a pattern of $P$ in $T$. Now, since $\left(1, y_{1}\right)$, $\left(2, y_{2}\right), \cdots,\left(m, y_{m}\right)$ is an $r$-pattern of $Q$ in $T$, it follows that if $i$ and $j$ are integers, $1 \leqq i, j \leqq m$, such that $|i-j| \leqq 1$, then $\left|y_{i}-y_{j}\right| \leqq 1$. In particular, $\left|y_{x_{\sim}}-y_{x_{b}}\right| \leqq 1$ whenever $\left|x_{a}-x_{b}\right| \leqq 1$. Furthermore, since $\left(1, x_{1}\right),\left(2, x_{2}\right), \cdots,\left(n, x_{n}\right)$ is an $r$-pattern of $P$ in $Q,\left|x_{a}-x_{b}\right| \leqq 1$ whenever $|a-b| \leqq 1,1 \leqq a, b \leqq n$. It follows that $\left|y_{x_{a}}-y_{x_{b}}\right| \leqq 1$ whenever $|a-b| \leqq 1$. This is the condition that the pattern $\left(1, y_{x_{1}}\right),\left(2, y_{x_{2}}\right), \cdots,\left(n, y_{x_{n}}\right)$ of $P$ in $T$ be an $r$-pattern. We conclude that $P$ is a refinement of $T$ and that the relation "refinement" is transitive.

If $P$ is a normal refinement of $Q$ and $Q$ is a normal refinement of $T$, then there is an $r$-pattern of the form $\left(1, x_{1}=1\right),\left(2, x_{2}\right), \cdots,\left(n, x_{n}=m\right)$ of $P$ in $Q$, and there is an $r$-pattern of the form $\left(1, y_{1}=1\right),\left(2, y_{2}\right), \cdots,\left(m, y_{m}=k\right)$ of $Q$ in $T$. Now, as in the previous case, the pattern $\left(1, y_{x_{1}}\right),\left(2, y_{x_{2}}\right), \cdots,\left(n, y_{x_{n}}\right)$ is an $r$-pattern of $P$ in $T$. Furthermore $y_{x_{1}}=y_{1}=1$ and $y_{x_{n}}=y_{m}=k$. But this is the condition that the $p$-chain $P$ be a normal refinement of the $p$-chain $T$. Hence the relation "normal refinement" is also transitive.

Finally, if $P$ is a principal refinement of $Q$ and $Q$ is a principal refinement of $T$, then there is an $r$-pattern $\left(1, x_{1}\right),\left(2, x_{2}\right), \cdots,\left(n, x_{n}\right)$ of $P$ in $Q$, an $r$-pattern $\left(1, y_{1}\right),\left(2, y_{2}\right), \cdots,\left(m, y_{m}\right)$ of $Q$ in $T$, and furthermore, $p_{i}=q_{x_{i}}$ for $i=1,3, \cdots, n$ and $q_{i}=t_{y_{i}}$ for $i=1,2, \cdots, m$. Again, $\left(1, y_{x_{1}}\right),\left(2, y_{x_{2}}\right), \cdots,\left(n, y_{x_{n}}\right)$ is an $r$-pattern of $P$ in $T$, and since also $p_{i}$ is equal to the link of $T$ with subscript $y_{x_{i}}, i=1,2, \cdots, n$, and each link of $T$ corresponds to at least one link of $P$, it follows that $P$ is a principal refinement of $T$. The relation "principal refinement," then, is also transitive. 
The next theorem has a prominent function in the combinatorial and refinemental relationships among $p$-chains. In particular it is an important preliminary theorem to the principal results of both $\S \S 3$ and 4 .

THEOREM 3.2. If a p-chain $P$ is a normal refinement of a p-chain $Q$ and $T$ is a p-chain which is a principal normal refinement of $Q$, then there is a p-chain $S$ such that $S$ is a principal normal refinement of $P$ and a normal refinement of $T$.

Proof. Let $P=\left(p_{1}, p_{2}, \cdots, p_{n}\right), Q=\left(q_{1}, q_{2}, \cdots, q_{m}\right)$ and $T=\left(t_{1}, t_{2}, \cdots, t_{k}\right)$, and let $\left(1, x_{1}=1\right),\left(2, x_{2}\right), \cdots,\left(n, x_{n}=m\right)$ be an $r$-pattern of $P$ in $Q$ and $\left(1, y_{1}=1\right)$, $\left(2, y_{2}\right), \cdots,\left(k, y_{k}=m\right), t_{i}=q_{y_{i}}, i=1,2, \cdots, k$, be an $r$-pattern of $T$ in $Q$. Since $P$ and $T$ are each normal refinements of $Q$, we may choose two increasing sequences of integers, $a_{1}, a_{2}, \cdots, a_{r}$ and $b_{1}, b_{2}, \cdots, b_{s}$ as follows. For the sequence $a_{1}, a_{2}, \cdots, a_{r}$ we first define $a_{1}=1$. Then, if there exist integers $j_{2}$ and $h_{2}$ such that $j_{2}<h_{2}<n$, $x_{j_{2}}=m$ and $x_{h_{2}}<m$, we define $a_{2}$ to be the first integer such that $x_{a_{2}}=m$. Otherwise we set $a_{2}=a_{r}=n$, and in this case the definition of the sequence is complete. In the former case let $j_{3}$ be the first integer greater than $a_{2}$ such that $x_{j_{3}}=m$ and for which there is an integer $h_{3}$ between $a_{2}$ and $j_{3}$ such that $x_{h_{3}}<m$. We define $a_{3}$ to be the first integer greater than $a_{2}$ such that $x_{a_{3}} \leqq x_{w}$ for $a_{2} \leqq w \leqq j_{3}$. If there are integers $j_{4}, h_{4}$ greater than $a_{3}$ such that $j_{4}<h_{4}, x_{j_{4}}=m$, and $x_{h_{4}}<m$, then we define $a_{4}$ to be the first integer greater than $a_{3}$ such that $x_{a_{4}}=m$. Otherwise we set $a_{4}=a_{r}=n$. It may be seen that this process can be continued and that the sequence so defined will be an increasing sequence of integers whose last member $a_{r}$ has the value $n$. We define the sequence $b_{1}, b_{2}, \cdots, b_{s}$ by replacing the letters $x, a, r$ and $n$, in the definition of the sequence $a_{1}, a_{2}, \cdots, a_{r}$, by the letters $y, b, s$ and $k$, respectively. Two other sequences of integers $c_{1}, c_{2}, \cdots, c_{s}$ and $d_{1}, d_{2}, \cdots, d_{r}$ are also chosen. The integer $c_{i}$ is defined to be the greatest integer such that $x_{c_{i}}=y_{b_{i}}$, $i=1,2, \cdots, s$, and $d_{i}$ is defined to be the greatest integer such that $y_{d_{i}}=x_{a_{i}}$, $i=1,2, \cdots, r$.

It is noted that the $p$-chain sums $P_{1}=P\left(a_{1}, a_{2}\right)+P\left(a_{2}, a_{3}\right)+\cdots+P\left(a_{r-1}, a_{r}\right)$ and $T_{1}=T\left(b_{1}, b_{2}\right)+T\left(b_{2}, b_{3}\right)+\cdots+T\left(b_{s-1}, b_{s}\right)$ are principal normal refinements of $P$ and $T$, respectively.

The theorem is now proved by induction on the number $k$ of links of $T$. If $k=1$, then $T$ and $Q$ are the same $p$-chain and we need only set $S=P$. The $p$-chain $S$ then has the required properties.

Suppose that the theorem has been established for all values of $k$ less than some integer $f, f>1$, and consider the case that $k=f$. It will be assumed that $y_{k-1}=m-1$, since otherwise the induction step is trivial. Two cases will be considered.

Case I. $s=2$. In this case the $p$-chain $T(1, k-1)$ is a principal normal refinement of $Q(1, m-1)$. Let $u$ be an integer such that $P(1, u)$ is maximal with respect to being a sub- $p$-chain of $P\left(a_{1}, a_{2}\right)$ and with respect to being a normal refinement of $Q(1, m-1)$. It is observed that the $p$-chain $T(k-1, k)$ and the 
$p$-chain $Q(m-1, m)$ are identical and that $P\left(u, a_{2}\right)$ is a normal refinement of this $p$-chain. Since $P(1, u)$ is a normal refinement of $Q(1, m-1)$, and since $T(1, k-1)$ is a principal normal refinement of $Q(1, m-1)$ having less than $k$ links, it follows by the induction hypothesis that there exists a $p$-chain $S_{11}$ such that $S_{11}$ is a principal normal refinement of $P(1, u)$ and a normal refinement of $T(1, k-1)$. It is easily seen that the $p$-chain sum $S_{1}=S_{11}+S_{12}$, where $S_{12}=P\left(u, a_{2}\right)$, is a defined $p$-chain sum, that $S_{1}$ is a principal normal refinement of $P\left(a_{1}, a_{2}\right)$, and $S_{1}$ is a normal refinement of $T$.

Now, the $p$-chain $P\left(a_{2}, a_{3}\right)$ is a normal refinement of $Q\left(m, x_{a_{3}}\right)$, and $T\left(k, d_{3}\right)$ is a principal normal refinement of $Q\left(m, x_{a_{3}}\right)$. Furthermore, $t_{d_{3}}$ is the first link of the $p$-chain $T\left(k, d_{3}\right)$ that corresponds to the last link of $Q\left(m, x_{a_{3}}\right)$ under the pattern of $T$ in $Q$, and $p_{a_{3}}$ is the first link of $P\left(a_{2}, a_{3}\right)$ that corresponds to the last link of $Q\left(m, x_{a_{3}}\right)$ under the pattern of $P$ in $Q$. This is similar to the situation just considered. It follows that there is a $p$-chain $S_{2}$ such that $S_{2}$ is a principal normal refinement of $P\left(a_{2}, a_{3}\right)$ and a normal refinement of $T\left(k, d_{3}\right)$. Similarly, there is a $p$-chain $S_{3}$, such that $S_{3}$ is a principal normal refinement of $P\left(a_{3}, a_{4}\right)$, and such that $S_{3}$ is a normal refinement of $T\left(d_{3}, k\right)$. Proceeding in this way we obtain $p$ chains $S_{1}, S_{2}, \cdots, S_{r-1}$, which are principal normal refinements of $P\left(a_{1}, a_{2}\right)$, $P\left(a_{2}, a_{3}\right), \cdots, P\left(a_{r-1}, a_{r}\right)$, respectively, and which are normal refinements of $T, T\left(k, d_{3}\right), T\left(d_{3}, k\right), \cdots, T\left(d_{r-1}, k\right)$. Then the $p$-chain sum $S=S_{1}+S_{2}+\cdots+S_{r-1}$ is defined, and $S$ is a principal normal refinement of $P_{1}$ and a normal refinement of $T_{1}$. It follows by Theorem 3.1 that $S$ is a $p$-chain of the desired type.

Case II. $s>2$. In this case, each of the $p$-chains $T\left(b_{1}, b_{2}\right), T\left(b_{2}, b_{3}\right), \cdots$, $T\left(b_{s-1}, b_{s}\right)$ has less than $k$ links. Now these $p$-chains are respectively principal normal refinements of $Q(1, m), Q\left(m, y_{b_{3}}\right), \cdots, Q\left(y_{b_{s-1}}, y_{b_{s}}=m\right)$. In addition, the p-chains $P, P\left(n, c_{3}\right), P\left(c_{3}, n\right), \cdots, P\left(c_{s-1}, n\right)$ are normal refinements of $Q(1, m)$, $Q\left(m, y_{b_{3}}\right), Q\left(y_{b_{3}}, m\right), \cdots, Q\left(y_{b_{s-1}}, m\right)$, respectively. Then, by the induction hypothesis, there exist $p$-chains $S_{1}, S_{2}, S_{3}, \cdots, S_{s-1}$ which are principal normal refinements of $P, P\left(n, c_{3}\right), P\left(c_{3}, n\right), \cdots, P\left(c_{s-1}, n\right)$, respectively, and which are normal refinements of $T\left(b_{1}, b_{2}\right), T\left(b_{2}, b_{3}\right), \cdots, T\left(b_{s-1}, b_{s}\right)$, respectively. Clearly, $S$ $=S_{1}+S_{2}+\cdots+S_{s-1}$ is a defined $p$-chain, and $S$ is a principal normal refinement of $P$ and a normal refinement of $T$. This completes the proof.

THEOREM 3.3. If a p-chain $P$ is a normal refinement of a p-chain $Q$, then there is a principal normal refinement $T$ of $P$ such that $T$ is crooked in $Q$ and is a normal refinement of $Q$.

Proof. Let $P=\left(p_{1}, p_{2}, \cdots, p_{n}\right), Q=\left(q_{1}, q_{2}, \cdots, q_{m}\right)$ and let $\left(1, x_{1}=1\right)$, $\left(2, x_{2}\right), \cdots,\left(n, x_{n}=m\right)$ be an $r$-pattern of $P$ in $Q$. We now define the $p$-chain $T=\left(t_{1}, t_{2}, \cdots, t_{k}\right)$ inductively with respect to the number, $n$, of links of $P$.

First, if $n=1$, then we set $T=P$ and observe that requirements of Definition 9 for $T$ in $P$ are vacuously satisfied. Next assume that for all values of $n$ less than some integer $s, s>1, T$ has been chosen to be a principal normal refinement of $P$, 
crooked in $P$, and consider the case that $n=s$. In this case, each of the $p$-chains $P(1, n-1), P(n-1,2)$ and $P(2, n)$ has less than $s$ links and we may choose $p$-chains $T_{1}, T_{2}$ and $T_{3}$ which are principal normal refinements crooked in $P(1, n-1), P(n-1,2)$ and $P(2, n)$, respectively. We then define $T$ to be the $p$ chain sum $T_{1}+T_{2}+T_{3}$. This completes the definition of the $p$-chain $T$ such that $T$ has an $r$-pattern in $P$ satisfying the requirements of Definitions 7, 8 and 9.

It remains to show that $T$ is crooked in $Q$. To do this, suppose that $t_{a}$ and $t_{b}$ are links of the $p$-chain $T$ and let these links be chosen so that $a<b$ and $\left|x_{y_{-}}-x_{y_{b}}\right|>2$. Then $\left|y_{a}-y_{b}\right|>2$ and, since $T$ is crooked in $P$, the sub- $p$-chain $T(a, b)$ of $T$ contains links $t_{r}$ and $t_{s}$ such that $r<s$ and we have $\left|y_{r}-y_{b}\right|=1$ and $\left|y_{s}-y_{a}\right|=1$. It follows that $\left|x_{y_{r}}-x_{y_{b}}\right| \leqq 1$ and $\left|x_{y_{s}}-x_{y_{n}}\right| \leqq 1$. Hence, there are links $t_{u}$ and $t_{v}$ of $T(a, b)$ such that $u<v,\left|x_{y}-x_{y_{b}}\right|=1$, and $\left|x_{y_{v}}-x_{y_{a}}\right|=1$. We conclude that $T$ is a principal normal refinement of $P, T$ is a normal refinement of $Q$, and $T$ is crooked in $Q$.

The following theorem, in addition to being a preliminary theorem to the characterizations of the continuous images of the pseudo-arc, is of some interest in itself. It is of direct use in investigating which classes of compact metric continua fail to be contained in the class of continuous images of the pseudo-arc.

THEOREM 3.4. If a continuum $H$ is a continuous image of the pseudo-arc and $x$ is a point of $H$, then there is a sequence of p-chains associated with $H$ such that the first link of each p-chain of the sequence contains $x$.

Proof. Let $M$ denote the pseudo-arc, let $f$ be a continuous mapping of $M$ onto $H$, and let $p$ be a point of $M$ such that $f(p)=x$. Now, by Theorem 10 [1], the continuum $M$ is indecomposable and we may choose a point $q$ from a composant of $M$ which does nor contain $p$. It then follows from the proof of Theorem 1 of [2] that $M$ is chainable between $p$ and $a$. Hence we may choose a sequence of chains $D_{1}, D_{2}, D_{3}, \cdots$ in $M$, considered as space, such that for each positive integer $i$, (1) $D_{i+1}$ is a refinement of $D_{i}$, (2) the diameter of each link of $D_{i}$ is less than $1 / i$, (3) the closure of each link of $D_{i+1}$ is a subset of some link of $D_{i}$, and (4) the union of the elements of $D_{i}$ is $M$.

For each positive integer $i$, let $D_{i}$ be the chain $\left(d_{i 1}, d_{i 2}, \cdots, d_{i n_{i}}\right)$ and let $f\left(D_{i}\right)$ denote the $p$-chain $\left(f\left(d_{i 1}\right), f\left(d_{i 2}\right), \cdots, f\left(d_{i n_{i}}\right)\right)$. We will now show that there is a sub-sequence of the sequence of $p$-chains $f\left(D_{1}\right), f\left(D_{2}\right), f\left(D_{3}\right), \cdots$ which is associated with $H$. To do this, we first note, since $M$ is compact, that the continuous mapping $f$ is uniformly continuous and hence, by property (2) above, we may choose a subsequence $f\left(D_{k_{1}}\right), f\left(D_{k_{2}}\right), f\left(D_{k_{3}}\right), \cdots$ of the sequence $f\left(D_{1}\right), f\left(D_{2}\right), f\left(D_{3}\right), \cdots$ such that, for each positive integer $i$, the diameter of each link of $f\left(D_{k_{i}}\right)$ is less than $1 / i$. Now, for $i=1,2,3, \cdots, D_{k_{i+1}}$ is a refinement of $D_{k_{i}}$ and, in addition, $D_{k_{i}}$ and $D_{k_{i+1}}$ are chains from $p$ to $q$ such that, by property (3), the closure of each link of $D_{k_{i+1}}$ is a subset of some link of $D_{k_{i}}$. Hence we may choose a pattern $\pi(i, i+1)$ of $D_{k_{i+1}}$ in $D_{k_{i}}$ which associates the first and last links of $D_{k_{i+1}}$ with the first and last 
links of $D_{k_{t}}$, respectively, and which associates each link of $D_{k_{i+1}}$ with a link of $D_{k_{i}}$ which contains its closure. This pattern $\pi(i, i+1)$ is clearly an $r$-pattern of $f\left(D_{k_{i+1}}\right)$ in $f\left(D_{k_{i}}\right)$ and from the nature of $\pi(i, i+1)$ it follows that $f\left(D_{k_{i+1}}\right)$ is a normal refinement of $f\left(D_{k_{i}}\right)$ and that, furthermore, if the link $f\left(d_{k_{i}}\right)$ of $f\left(D_{k_{i}}\right)$ corresponds to the link $f\left(d_{k_{i+1} r}\right)$ of $f\left(D_{k_{i+1}}\right)$, then $\overline{f\left(d_{k_{i+1} r}\right)}=\overline{f\left(d_{k_{i+1} r}\right)} \subset f\left(d_{k_{i} s}\right)$. Finally, from property (4) above, it is clear that the union of the elements of $f\left(D_{k_{i}}\right)$ is $H$, for each positive integer $i$. Thus, the sequence of $p$-chains $f\left(D_{k_{1}}\right)$, $f\left(D_{k_{2}}\right), f\left(D_{k_{3}}\right), \cdots$ satisfies each of the requirements of Definition 10 with respect to $H$ and each $p$-chain of this sequence has $x$ in its first element.

The theorem which follows constitutes the first characterization of the continuous images of the pseudo-arc and is one of the principal results of this section. In view of the factors mentioned in footnote 1 , we shall give a condensed form of the proof of this theorem. In this proof, as well as in the development of the later sections of this paper, we shall make strong use of the combinatorial and refinemental properties of $p$-chains rather than special properties of chain coverings of the pseudo-arc.

THEOREM 3.5. In order that a continuum $H$ be a continuous image of the pseudo-arc it is necessary and sufficient that $H$ be p-chainable.

Proof of necessity. This follows from Theorem 3.4.

Proof of sufficiency. Let $H$ be a $p$-chainable continuum and let $P_{1}, P_{2}, P_{3}, \cdots$ be a sequence of $p$-chains associated with $H$. We shall define two related sequences of $p$-chains $T_{1}, T_{2}, T_{3}, \cdots$ and $D_{1}, D_{2}, D_{3}, \cdots$ having the following properties: for each positive integer $n$, (1) $T_{n}$ is a principal normal refinement of $P_{n}$, (2) $D_{n}$ is a corresponding chain of open discs in the plane having the same number of links as $T_{n}$, and, if $n$ is greater than 1 , then (3) $T_{n}$ is crooked in $T_{n-1}, D_{n}$ has the same pattern in $D_{n-1}$ as the $r$-pattern of $T_{n}$ in $T_{n-1}$, each link of $D_{n}$ has diameter less than $1 / n$ and the closure of each link of $D_{n}$ is contained in the corresponding link of $D_{n-1}$. In the case that $n=1$, it is observed that these requirements may readily be satisfied. Next assume for each positive integer $n$ less than some integer $k, k>1$, that the requirements of this induction statement have been satisfied and consider the case that $n=k$.

First, by Theorem 3.2, we note that there is a $p$-chain $S_{k}$ which is a principal normal refinement of $P_{k}$ and a normal refinement of $T_{k-1}$. Furthermore, by Theorem 3.3, there is a principal normal refinement $T_{k}$ of $S_{k}$ such that $T_{k}$ is crooked in $T_{k-1}$. Hence, noting Theorem 3.1, $T_{k}$ is a principal normal refinement of $\boldsymbol{P}_{\boldsymbol{k}}$ which is crooked in $T_{k-1}$. In addition, we may assume without loss in generality that $T_{k}$ has a sufficient number of links so that we can choose a chain $D_{k}$ of open discs in the plane which fulfills the remaining conditions of property (3) for $n=k$. It follows that $T_{k}$ and $D_{k}$ satisfy the requirements of the induction hypothesis and the definition of the sequences of $p$-chains $T_{1}, T_{2}, T_{3}, \cdots$ and $D_{1}, D_{2}, D_{3}, \cdots$ is 
complete. It is noted, from $[2, \S 2]$, that the intersection of the sets of points of the chains $D_{1}, D_{2}, D_{3}, \cdots$ is a pseudo-arc $M$.

We now define a continuous mapping $f$ of $M$ onto $H$. To do this, ler $x$ be a point of $M$ and let the links $d_{1 r_{1}}$ of $D_{1}, d_{2 r_{2}}$ of $D_{2}, d_{3 r_{3}}$ of $D_{3}, \cdots$ be a sequence of open sets closing down on $x$ such that, for each positive $i, d_{i+1 r_{i+1}}$ corresponds to $d_{i r_{i}}$ under the pattern of $D_{i+1}$ in $D_{i}$. Then, by conditions (c) and (d) of Definition 10 , the intersection $\bigcap_{i=1}^{\infty} t_{i r_{i}}$ exists and is a single point. We define the mapping $f$ by setting $f(x)=\bigcap_{i=1}^{\infty} t_{i r_{i}}$. To see that $f$ is uniquely defined, suppose that $d_{1 r_{1}}$, $d_{2 r_{2}}, d_{3 r_{3}}, \cdots$ and $d_{1 s_{1}}, d_{2 s_{2}}, d_{3 s_{3}}, \cdots$ are two sequences of links closing down on $x$ in the above described manner. Then, $\left|r_{i}-s_{i}\right| \leqq 1, i=1,2,3, \cdots$, so that $t_{i r_{i}} \cup t_{i s_{i}}$ has diameter less than $2 / i$ and hence $\bigcap_{i=1}^{\infty}\left(t_{i r_{i}} \cup t_{i s_{i}}\right)=\bigcap_{i=1}^{\infty} t_{i r_{i}}=\bigcap_{i=1}^{\infty} t_{i s_{i}}$. To prove that $f$ is a continuous mapping of $M$ into $H$, let $g$ be any open set in $H$ and let $k$ be an integer such that $g$ contains three consecutive links $t_{k c-1}, t_{k c}$ and $t_{k c+1}$ of $T_{k}$. Now $D_{k}$ is a chain and if $d_{1 r_{1}}, d_{2 r_{2}}, d_{3 r_{3}}, \cdots$ is a sequence which closes down on a point of $d_{k c}$, then one of the links $d_{k c-1}, \mathrm{~d}_{k c}$ and $d_{k c+1}$ is a member of this sequence. Thus $d_{k c} \cap M$ which is open in $M$ is mapped into $t_{k c-1} \cup t_{k c} \cup t_{k c+1}$ and hence into $g$ under the mapping $f$. It follows that $f$ is continuous and that $f(M)$ is everywhere dense in $M$. But $M$ is compact so that the continuous transformation $f$ is a mapping of $M$ onto $H$, and the proof is complete.

The following theorem indicates an analogy between Theorem 3.5 and the Hahn-Mazurkiewicz characterization of the continuous images of the arc. It may be seen that it follows easily from the proof of the Hahn-Mazurkiewicz theorem $[5 ; 8]$.

THEOREM 3.6. In order that a compact continuum $H$ be locally connected it is necessary and sufficient that $H$ be p-chainable with p-chains whose links are connected open sets.

With this formulation of the definition of local connectedness, the characterization of the continuous images of the pseudo-arc of Theorem 3.5 and the HahnMazurkiewicz characterization of the continuous images of the arc differ only in that the links of the $p$-chains involved in the latter characterization are required to be connected open sets.

In the next and final theorem of this section we establish an alternative characterization of the continuous images of the pseudo-arc which, while it has certain disadvantages in investigating the combinatorial properties among these continua, involves a somewhat closer analogy to the Hahn-Mazurkiewicz theorem than does Theorem 3.5.

THEOREM 3.7. A necessary and sufficient condition that a compact continuum $H$ be a continuous image of the pseudo-arc is that $H$ be p-chainable with $p$ chains whose links are open sets.

Proof of necessity. Let $H$ be a continuous image of the pseudo-arc and, for 
convenience, let $H$ be considered as space. Then, by Theorem 3.5, there is a sequence of $p$-chains $P_{1}, P_{2}, P_{3}, \cdots$ associated with $H$ and we may without loss in generality assume that, for each positive integer $n$, each link of $P_{n}$ has diameter less than $1 / 2 n$. We now choose a sequence of $p$-chains $T_{1}, T_{2}, T_{3}, \cdots$ associated with $H$ such that, for each positive integer $n, T_{n}$ has the same number of links as $P_{n}, T_{n+1}$ has a pattern in $T_{n}$ which is the same as the $r$-pattern of $P_{n+1}$ in $P_{n}$, and each link of $T_{n}$ is an open set. To do this, let $P_{n}=\left(p_{n 1}, p_{n 2}, \cdots, p_{n r_{n}}\right)$ and let $T_{n}=\left(t_{n 1}, t_{n 2}, \cdots, t_{n r_{n}}\right)$ where $t_{n i}, i=1,2, \cdots, r_{n}$, is the set of all points which have distance less than $1 / 4 n$ from the link $p_{n i}$ of $P$. With this definition of $T_{n}$, it is clear that $T_{n}$ is a $p$-chain whose links are open sets. Furthermore, for each positive integer $n$, the union of the elements of $T_{n}$ is $H$, each link of $T_{n}$ has diameter less than $1 / n, T_{n+1}$ has a pattern in $T_{n}$ which is the same as the $r$-pattern of $P_{n+1}$ in $P_{n}$, and, under this pattern, the closure of each link of $T_{n+1}$ is a subset of the corresponding link of $T_{n}$. Thus $T_{1}, T_{2}, T_{3}, \cdots$ is a sequence of $p$-chains associated with $H$ such that each link of each $p$-chain of this sequence is an open set and the proof is complete.

Proof of sufficiency. This follows from Theorem 3.5.

4. Chainable continua. In this section it will be shown that every chainable continuum is a continuous image of the pseudo-arc. The proof will primarily depend on Theorem 3.2 and the characterization of the continuous images of the pseudo-arc stated in Theorem 3.5.

THEOREM 4.1. Every chainable continuum is a continuous image of the pseudo-arc.

Before proceeding to give a proof of this theorem, it will be convenient to establish two lemmas.

Lemma 4.1.1. If $P$ and $Q$ are $p$-chains such that $P$ is a refinement of $Q$ and each link of $Q$ corresponds to at least one link of $P$, then there is a p-chain $T$ such that $T$ is a principal refinement of $P$ and a normal refinement of $Q$.

Proof. Let the $p$-chain be $P(1, n)$ and let $p_{s}$ and $p_{t}$ be links of $P$ which correspond to the first and last links of $Q$, respectively, under the $r$-pattern of $P$ in $Q$. We will then define $T$ to be the $p$-chain sum $P(s, 1)+P(1, n)+P(n, t)$. It is clear that the $p$-chain $T$ then has the required properties.

LEMMA 4.1.2. If $P, Q$ and Tare p-chains such that $T$ is a principal refinement of $Q$ and $P$ is a refinement of $Q$ in which each link of $Q$ corresponds to at least one link of $P$, then there is a p-chain $S$ such that $S$ is a principal refinement of $P$ and $S$ is a refinement of $T$ in which each link of $T$ corresponds to at least one link of $S$.

Proof. First, from the preceding lemma we observe that there exist $p$-chains $P_{1}$ and $T_{1}$ which are principal refinements of $P$ and $T$, respectively, and which 
are each normal refinements of the $p$-chain $Q$. In addition, from Theorem 3.1, $T_{1}$ is also a principal refinement of $Q$. It follows, by Theorem 3.2, that there is a $p$-chain $S$ such that $S$ is a principal normal refinement of $P_{1}$ and a normal refinement of $T_{1}$. Moreover, by Theorem 3.1, $S$ is then a refinement of $T$ and a principal refinement of $P$. Finally, since $S$ is a normal refinement of $T_{1}$ and $T_{1}$ is a principal refinement of $T$, each link of $T$ corresponds to at least one link of $S$. Thus, the $p$-chain $S$ has the required properties and the proof is complete.

Proof of Theorem 4.1. Let $H$ be a chainable continuum and, for convenience, let $H$ be considered as space. Then there is a sequence of chains $D_{1}, D_{2}, D_{3}, \cdots$ such that for each positive integer $i$, (1) the set of points of $D_{i}$ is $H$, (2) each link of $D_{i}$ has diameter less than $1 / i,(3)$ no link of $D_{i}$ is a subset of any other link of $D_{i}$, and (4) there is an $r$-pattern of $D_{i+1}$ in $D_{i}$ such that the closure of each link of $D_{i+1}$ is a subset of the corresponding link of $D_{i}$. We now construct a corresponding sequence of $p$-chains $P_{1}, P_{2}, P_{3}, \cdots$ associated with $H$. First we define the $p$-chain $P_{1}$ to be the chain $D_{1}$. Then we observe, from property (3), above, and the connectedness of $H$, that each link of $D_{1}$ corresponds to at least one link of $D_{2}$, and therefore, by Lemma 4.1.1 we may choose $P_{2}$ to be a $p$-chain which is a principal refinement of $D_{2}$ and a normal refinement of $P_{1}$. Next, since each link of $D_{2}$ corresponds to at least one link of $D_{3}$, by Lemma 4.1.2 there is a $p$-chain $S_{3}$, such that $S_{3}$ is a principal refinement of $D_{3}$, and $S_{3}$ is a refinement of $P_{2}$ in which each link of $P_{2}$ corresponds to at least one link of $S_{3}$. It then follows by Lemma 4.1.1 that we may choose $P_{3}$ to be a principal refinement of $S_{3}$ and hence of $D_{3}$, and a normal refinement of $P_{2}$. Proceeding in this manner, we obtain a sequence of $p$-chains, $P_{1}, P_{2}, P_{3}, \cdots$ which are principal refinements of the chains $D_{1}, D_{2}, D_{3}, \cdots$, respectively, and which have the property that each is a normal refinement of the $p$-chain (if any) that precedes it in the sequence. This is clearly a sequence of $p$-chains associated with $H$ and, by Theorem 3.5, we conclude that $H$ is a continuous image of the pseudo-arc.

Corollary 4.1.1. The class of continuous images of the pseudo-arc and the class of continuous images of all chainable continua are identical.

5. Nonexistence of a local characterization of the continuous images of the pseudo-arc. In $\$ 3$ we have established two characterizations of the continuous images of the pseudo-arc and have shown that, if the definition of local-connectedness is suitably reformulated as a global property, then these characterizations are analogous to the Hahn-Mazurkiewicz characterization of the continuous images of the arc $[5 ; 8]$. However, since an important characteristic of this Hahn-Mazurkiewicz theorem is the identification of the continuous images of the arc by a simple local property, the question arises, "Does there exist a local topological property which characterizes all continuous images of the pseudoarc?"' In this section we shall show that this question has a negative answer.

A number of additional special terms, some of which will also be of significance in $\S 6$, will be needed. 
DEFINITION 11. If $S$ is a metric space and $x, y$ are points such that there is a shortest arc from $x$ to $y$ in $S$, then the arc-distance between $x$ and $y$ will be defined to be equal to the length of such a shortest arc. A unique arc from $x$ to $y$ in $S$ will be denoted by $[x, y]$.

Definition 12. If $M$ is a set such that there is a defined arc distance between each two points in $M$, then we define the arc-diameter of a subset of $M$, and the arc-distance between two sets in $M$ in the usual way in terms of arc-distance between points.

DefintTION 13. An $\varepsilon$-sequence of points in an arc $[x, y]$ will be a finite sequence of points, lying in $[x, y]$, such that the arc-distance between adjacent members of the sequence is less than $\varepsilon$.

Next, we formalize the definition of "local topological property" which will be used in this section. This expression appears to be not yet completely standardized. However, the definition to be given will be one of the more natural forms in general use, and will include all of the properties normally referred to in this category.

Definition 14. A space $S$ will be said to have a local topological property $\pi$ at a point $x$ if for every open set $U$ containing $x$ there is an open set $V$ such that $V$ contains $x$ and lies in $U$, and either $V$ or the closure of $V$ has the topological property $\pi$. The space $S$ will be said to have the local topological property $\pi$ if $S$ has property $\pi$ at each of its points.

Finally, we define a relationship between two topological spaces which essentially requires that any local topological property of one space necessarily must be a local topological property of the second space.

Definition 15. Two topological spaces $H$ and $K$ will be said to be locally homeomorphic if (1) for every point $x$ of $H$ and every open set $U$ containing $x$, there is an open set $V$ such that $V$ contains $x$ and lies in $U$ and $V$ is homeomorphic to an open set in $K$, and (2) a similar statement can be made with the roles of $H$ and $K$ interchanged.

We now proceed to formulate the theorem which will be the principal result of this section.

THEOREM 5.1. There does not exist a local topological property which characterizes all continuous images of the pseudo-arc.

In order to establish this theorem we shall consider a second result, Theorem 5.2. This latter theorem will show that there does not exist a local topological property of a continuum at any one of its points or on any collection of its points, which characterizes it as a continuous image of the pseudo-arc. In particular Theorem 5.1 will be a direct consequence of Theorem 5.2.

THEOREM 5.2. There exist locally homeomorphic compact metric continua $H$ and $K$ such that $H$ is a continuous image of the pseudo-arc, but $K$ is not a continuous image of the pseudo-arc. 
Proof. Since this proof will involve a rather complicated development it will be convenient to make a preliminary outline of the steps we shall follow.

First, the compact metric continua $H$ and $K$ will be defined. These continua will be considered as spaces and, for the reference needs of the later stages of the proof, will be described in precise detail. However, the essential features of these continua, which will be sufficient for the needs of the early stages of the proof, will be more readily seen from the accompanying figures. Then, we shall give arguments to show, in turn, that (a) $H$ is a continuous image of the pseudo-arc, and that (b) $H$ and $K$ are locally homeomorphic continua. Finally, the most difficult part of the proof will be considered. Specifically, we shall use Theorem 3.4 and the concepts introduced in the preceding segments of this section to establish that (c) the continuum $K$ is not a continuous image of the pseudo-arc.

Definition of $H$. In terms of plane cartesian co-ordinates the continuum $H$ will be defined to be the closure of the ray

$$
\begin{aligned}
& A=\bigcup_{n=4}^{\infty}\left[\left\{(u, v): u= \pm 2^{-n}, 2^{-n} \leqq v \leqq 2+2^{-n}\right\}\right. \\
& \cup\left\{(u, v):-2^{-n} \leqq u \leqq 2^{-n}, v=2+2^{-n}\right\} \\
& \cup\left\{(u, v):-2-2^{-n} \leqq u \leqq-2^{-n}, 2^{-n} \leqq u \leqq 2+2^{-n}-2^{-(n+2)}|(\sin n \pi / 2)|, v=2^{-n}\right\} \\
& \cup\left\{(u, v):-2-2^{-n} \leqq u \leqq 2+2^{-n}-2^{-(n+2)}|\cos (n \pi / 2)|, v=-2^{-n}\right\} \\
& \cup\left\{(u, v): u=-2-2^{-n},-2^{-n} \leqq v \leqq 2^{-n}\right\} \\
& \cup\left\{(u, v): 2+2^{-(n+1)}, \leqq u \leqq 2+3 \cdot 2^{-(n+2)}, v=0\right\} \\
& \cup\left\{(u, v): u=2+2^{-n}-2^{-(n+2)}|\sin (n \pi / 2)|, 0 \leqq v \leqq 2^{-n}\right\} \\
& \left.\cup\left\{(u, v): u=2+2^{-n}-2^{-(n+2)}|\cos (n \pi / 2)|,-2^{-n} \leqq v \leqq 0\right\}\right] .
\end{aligned}
$$

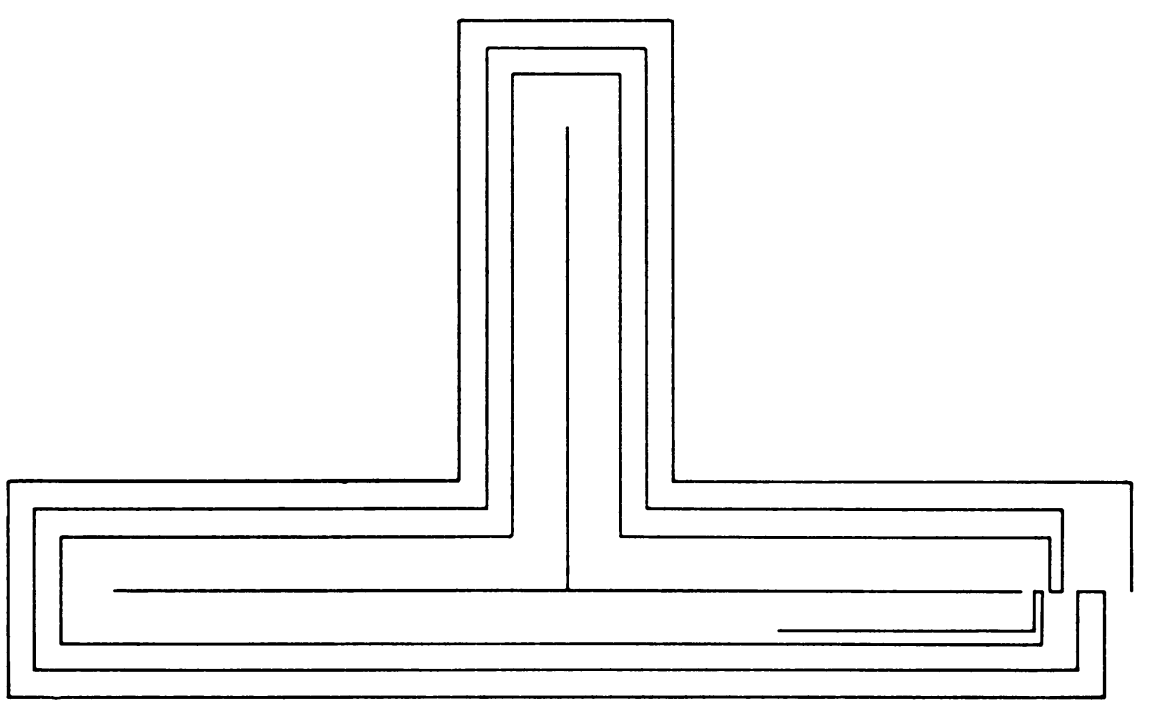

FIGURE 1 
Definition of $K$. In terms of plane cartesian co-ordinates the continuum $K$ will be defined to be the closure of the ray

$$
\begin{aligned}
B= & \left\{(w, z): w=2+2^{-4}, 0 \leqq z \leqq 2^{-4}\right\} \\
& \cup\left[\bigcup _ { n = 4 } ^ { \infty } \left(\left\{(w, z): 2^{-n} \leqq w \leqq 2+2^{-n},-2-2^{-n} \leqq w \leqq-2^{-n}, z=2^{-n}\right\}\right.\right. \\
& \cup\left\{(w, z): w= \pm 2^{-n}, 2^{-n} \leqq z \leqq 2+2^{-n}\right\} \\
& \cup\left\{(w, z):-2^{-n} \leqq w \leqq 2^{-n}, z=2+2^{-n}\right\} \\
& \cup\left\{(w, z): w=-2-2^{-n},-2^{-n} \leqq z \leqq 2^{-n}\right\} \\
& \cup\left\{(w, z):-2-2^{-n} \leqq w \leqq 2+2^{-(n+1)}, z=-2^{-n}\right\} \\
& \left.\left.\cup\left\{(w, z): w=2+2^{-(n+1)},-2^{-n} \leqq z \leqq 2^{-(n+1)}\right\}\right)\right] .
\end{aligned}
$$

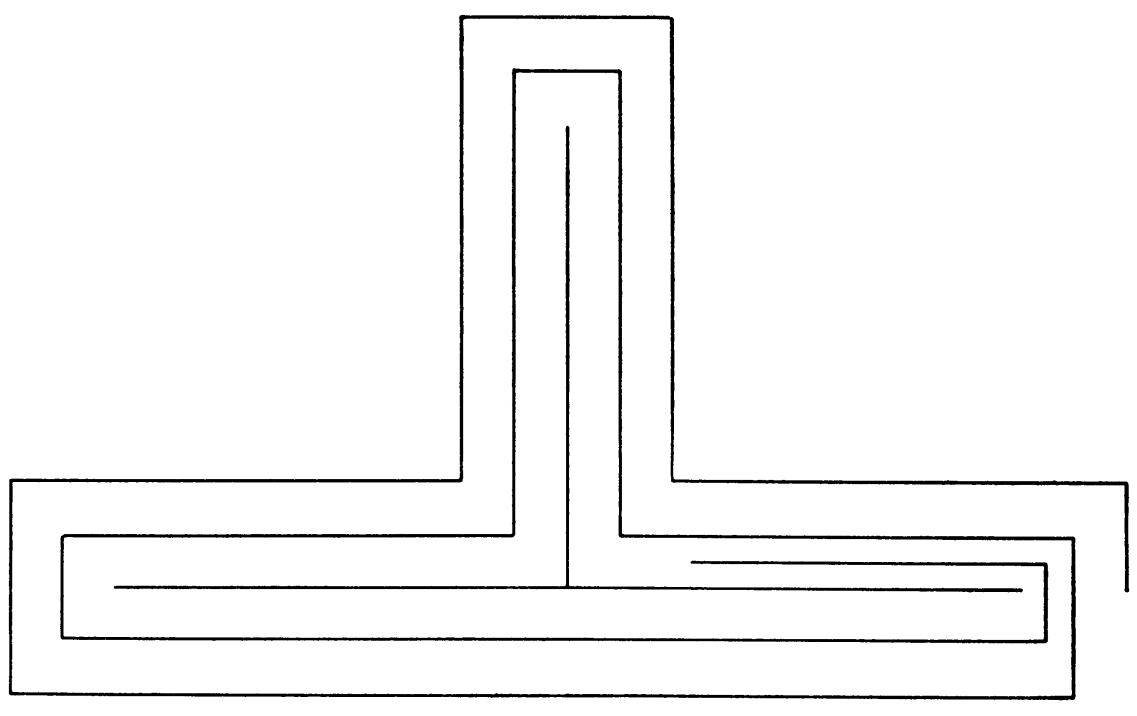

FIGURE 2

(a) Proof that $H$ is a continuous image of the pseudo-arc. If $C$ is the continuum which is the closure of the ray $R=\{(x, y): y=\sin 1 / x, 0<x \leqq 1\}$, there is a continuous mapping of the arc $C-R$ onto the simple triad $H-A$ which can be extended in a natural manner to a continuous mapping of $C$ onto $H$. Furthermore, $C$ is a chainable continuum. Hence, by Theorem 4.1, it follows that $H$ is a continuous image of the pseudo-arc.

(b) Proof that the compact continua $H$ and $K$ are locally homeomorphic. First, we observe that each point of $H$, other than the point $h=(u=2, v=0)$, is contained in a neighborhood which is homeomorphic to a neighborhood of $K$ 
under the identity mapping. Similarly, each point of $K$ other than the point $k=(w=2, z=0)$ is contained in a neighborhood which is homeomorphic to a neighborhood of $H$ under the identity mapping. Now consider the neighborhoods $N_{h}$ in $H$ with center $h$ and radius $r, r<2$, and $N_{k}$ in $K$ with center $k$ and the same radius $r$. We shall complete the proof of this section of the theorem by describing a homeomorphism $f$ of $N_{h}$ onto $N_{k}$. First, $f$ will be required to map the component of $N_{h}$ which contains $h$ onto the component of $N_{k}$ which contains $k$, in an identical manner. Then, if $C_{n}$ is the component of $N_{h}$ which is $n$th in the decreasing order of distances of the components of $N_{h}$ from the point $h$, and $D_{n}$ is the similarly determined component of $N_{k}$, we shall define $f$ over the part $C_{n}$ of its domain to be a particular homeomorphism of $C_{n}$ onto $D_{n}$. Specifically, let $e_{1}$ be the end-point of the arc $\bar{C}_{n}$ having smallest abscissa, let $e_{2}$, be the point of $\bar{C}_{n}$ which has abscissa equal to 2 and is closest to $e_{1}$, let $e_{3}$ be the point of $\bar{C}_{n}$ which has abscissa equal to 2 and is farthest from $e_{1}$, and finally let $e_{4}$ be the remaining endpoint of $\bar{C}_{n}$. In addition, let $g_{1}, g_{2}, g_{3}$ and $g_{4}$ be the similarly chosen points of $\bar{D}_{n}$. Then we shall define a transformation of $\bar{C}_{n}$ onto $\bar{D}_{n}$ by mapping $\left[e_{1}, e_{2}\right]$ onto $\left[g_{1}, g_{2}\right]$ in an order preserving and arc-distance preserving manner, mapping $\left[e_{2}, e_{3}\right]$ onto $\left[g_{2}, g_{3}\right]$ in an order preserving manner and mapping $\left[e_{3}, e_{4}\right]$ onto $\left[g_{3}, g_{4}\right]$ in an order preserving and arc-distance preserving manner. The restriction of this transformation to the domain $C_{n}$ is then a homeomorphism. The transformation $f$ over the part $C_{n}$ of its domain will be defined to be this homeomorphism. Thus, the definition of the mapping $f$ of $N_{h}$ onto $N_{k}$ is complete. We observe that $f$ is clearly continuous at all points of $N_{h}$ at which this set is locally connected. Furthermore, if $x$ is a point of $N_{h}$ at which $N_{h}$ fails to be locally connected and $x_{1}, x_{2}, x_{3}, \cdots$ is any sequence of points of $N_{h}$ which converges to $x$ then it is easily verified that $f\left(x_{1}\right), f\left(x_{2}\right), f\left(x_{3}\right), \cdots$ converges to the appropriate point $f(x)$ of $N_{k}$. Since, in addition, corresponding statements can be made for the inverse transformation $f^{-1}$ with respect to its domain $N_{k}$, we conclude that $f$ is a homeomorphism. This completes the proof that the compact continua $A$ and $K$ are locally homeomorphic.

(c) Proof that $K$ is not a continuous image of the pseudo-arc. For the purpose of investigating the continuum $K$ we choose a sequence of reference points $a_{0}, a_{1}, a_{2}, \cdots$ where $a_{0}=\left(w=2+2^{-4}, z=0\right)$ and is referred to as the first point of the ray $B$, the points $a_{0}, a_{2}, a_{4}, \cdots$ are the successive points of intersection of $B$ with the union of the $w$-axis and the positive $z$-axis, and $a_{1}, a_{3}, a_{5}, \cdots$ are the mid-points of the arcs $\left[a_{0}, a_{2}\right],\left[a_{2}, a_{4}\right],\left[a_{4}, a_{6}\right], \cdots$ respectively. We now proceed to show that $K$ is not a continuous image of the pseudo-arc.

Suppose, on the contrary, that the continuum $K$ is a continuous image of the pseudo-arc. Then, by Theorem $3.5, K$ is $p$-chainable and, by Theorem 3.4, we may choose a sequence of $p$-chains associated with $K$ such that the first link of each $p$-chain of the sequence contains the point $a_{0}$. Let $P=\left(p_{1}, p_{2}, \cdots, p_{n}\right)$ and $Q=\left(q_{1}, q_{2}, \cdots, q_{m}\right)$ be two $p$-chains of such a sequence and let these $p$-chains be 
chosen so that $P$ is a refinement of $Q$ with an $r$-pattern $\left(1, x_{1}=1\right),\left(2, x_{2}\right), \cdots$, $\left(n, x_{n}\right)$ in $Q$, each link of $P$ has diameter less than $\varepsilon=2^{-m}$, and each link of $Q$ has diameter less than $1 / 4$. For the purposes of this argument we need to observe, since the elements of $Q$ form a cover for $K$, that $m$ is greater than 7 .

From Definition 10 and the choice of restriction of the diameters of the links of $\boldsymbol{P}$ it follows that the first link of $\boldsymbol{P}$ has arc-diameter less than $\varepsilon$ and, if $p_{\boldsymbol{r}}$ is the first link of $P$ with arc-distance less than $2 \varepsilon$ from $a_{m}$ then each link of the sub- $p$ chain $P(1, r)$ is contained in the arc $\left[a_{0}, a_{m}\right]$ and has arc-diameter less than $2 \varepsilon$. Hence, we may select a $2 \varepsilon$-sequence of points $c_{1}, c_{2}, \cdots, c_{r}$ in $\left[a_{0}, a_{m}\right]$ such that for each integer $i, 1 \leqq i \leqq r, c_{i}$ is an element of the link $p_{i}$ of $P$. With this sequence of points and a corresponding sequence of points to be chosen from the links of $Q$ it will be shown that $K$ is not a continuous image of the pseudo-arc. The sequence $d_{1}, d_{2}, \cdots, d_{s}$ of points of the links of $Q$ is defined by first setting $d_{1}=a_{0}$ and then, if $i \geqq 1$ and $d_{i}$ has been chosen, and there is a link of $P(1, r)$ which fails to correspond to a link of $Q(1, i)$ under the $r$-pattern of $P$ in $Q$, we define $d_{i+1}$ to be a point of the first such link of $P(1, r)$. Then, $d_{1}, d_{2}, \cdots, d_{s}$ is a 1 -sequence of points in $\left[a_{0}, a_{m}\right]$ and for each integer $j, 1 \leqq j \leqq s$, the point $d_{j}$ is contained in the link $q_{j}$ of the $p$-chain $Q$.

Now, the $r$-pattern of $P$ in $Q$ defines a natural correspondence between the points $c_{1}, c_{2}, \cdots, c_{r}$ of the links of $P(1, r)$ and the points $d_{1}, d_{2}, \cdots, d_{s}$ of the links of $Q(1, s)$. Furthermore under this $r$-pattern each point $c_{i}$ of $c_{1}, c_{2}, \cdots, c_{r}$ has distance less than $1 / 4$ from the corresponding point $d_{x_{i}}$ of $d_{1}, d_{2}, \cdots, d_{s}$.

For the next step in our proof, we shall prove by induction that, for each integer $i, 1 \leqq i \leqq r$, the point $c_{i}$ bas arc-distance less than 6 from $d_{x_{i}}$.

In the case that $i=1$ we need only observe that $c_{1}$ and $d_{x_{1}}=d_{1}$ are each points of the link $p_{1}$ of $P$ and $p_{1}$ has arc-diameter less than $\varepsilon$ which is less than 6 .

Next, assume that the induction statement has been established for all values of $i$ less than or equal to some integer $t, 1 \leqq t<r$, and consider the situation that $i=t+1$. We let $\left[a_{u}, a_{u+1}\right]$ be an arc containing $c_{t}$ and let $z$ be the midpoint of this arc. Two cases will now be considered.

In the first case, we suppose that $c_{t}$ is an element of whichever of the arcs $\left[a_{u}, z\right]$ and $\left[z, a_{u+1}\right]$ is nearest to the origin. Then, by the induction hypothesis, $c_{t}$ and $d_{x_{t}}$ have arc-distance apart less than 6 and, by the nature of the $r$-pattern, $c_{t}$ and $d_{x_{t}}$ have distance apart less than $1 / 4$. It is easily seen from the geometrical nature of $K$ that these two conditions can only obtain if $c_{t}$ and $d_{x_{t}}$ have arc-distance apart of less than $4 \frac{1}{2}$. But the arc-distance between $c_{t}$ and $c_{t+1}$ is less than $2 \varepsilon$ and the arc-distance between $d_{x}$ and $d_{x_{t+1}}$ is less than 1 . Hence, the arc-distance between $c_{t+1}$ and $d_{x_{t+1}}$ is less than 6 .

In the second case, we suppose that $c_{t}$ is an element of whichever of the two $\operatorname{arcs}\left[a_{u}, z\right]$ and $\left[z, a_{u+1}\right]$ is farthest from the origin. Here, the restrictions on the distance and arc-distance between the points $c_{t}$ and $d_{x_{t}}$ and the geometrical nature of $K$ imply that the arc-distance between $c_{t}$ and $d_{x_{t}}$ is less than 3 . Then, as in the 
previous case the arc-distance between the points $c_{t+1}$ and $d_{x_{t+1}}$ is less than 6 . This completes the induction.

Now, from this preceding induction result, we are able to obtain a contradiction to the initial hypothesis that $K$ is $p$-chainable by showing that $Q$ must have more than $m$ links. Specifically, we note that $c_{r}$ has arc-distance less than 4 from $a_{m}$ and $d_{x_{r}}$ has arc-distance less than 6 from $c_{r}$. Thus $a_{m}$ and $d_{x_{r}}$ have arc-distance apart of less than 7. But $d_{x_{r}}$ is a point of a 1-sequence of points $d_{1}, d_{2}, \cdots, d_{s}$, where $d_{1}=a_{0}$, in $\left[a_{0}, a_{m}\right]$, and the length of the arc $\left[a_{0}, a_{m}\right]$ is greater than $2 m$. It follows that the 1 -sequence of points $d_{1}, d_{2}, \cdots, d_{s}$ in $\left[a_{0}, a_{m}\right]$ has more than $2 m-7$ members and, since $m$ is greater than 7, we have the result that $s$ is greater than $m$. This involves the desired contradiction and we conclude that $K$ is not a continuous image of the pseudo-arc.

6. Continua which are not continuous images of the pseudo-arc. In $\$ 4$, we have shown that the pseudo-arc can be mapped continuously onto all chainable continua and it is observed that, of course, all locally connected compact metric continua are continuous images of the pseudo-arc. Furthermore, the pseudo-arc is highly complex $[2 ; 7 ; 10]$ and, thus, it might be conjectured that the principal generalizations of the class of chainable continua and the class of locally connected compact metric continua are also contained in the class of continuous images of the pseudo-arc. However, this is not the case and in this section we shall give examples to show that the classes of tree-like continua, arc-wise connected compact metric continua, semi-locally connected compact metric continua, and aposyndetic [6] compact metric continua, each fail to be contained in the class of continuous images of the pseudo-arc.

EXAMPLE 6.1. A tree-like plane continuum which is not a continuous image of the pseudo-arc.

The plane continuum $K$ which was discussed in Theorem 5.2, and represented in Figure 2, is clearly tree-like and is not a continuous image of the pseudo-arc.

EXAMPLE 6.2. An arc-wise connected, compact metric continuum $C$ which is not a continuous image of the pseudo-arc.

Definition of $C$. In terms of spherical co-ordinates, the continuum $C$ is defined to be the closure of the ray

$$
R=\left\{(r, \theta, \phi): r=2-2^{-\theta / 2 \pi}, \theta \geqq 4 \pi, \phi=\pi / 2\right\}
$$

together with the arc

$$
\begin{aligned}
A= & \{(r, \theta, \phi): r \sin \phi=2, \theta=0, \pi / 4 \leqq \phi \leqq \pi / 2\} \\
& \cup\left\{(r, \theta, \phi): r \sin \phi=2-2^{-4}, \theta=0, \pi / 4 \leqq \phi \leqq \pi / 2\right\} \\
& \cup\left\{(r, \theta, \phi): 2 \sqrt{ } 2 \geqq r \geqq\left(2-2^{-4}\right) \sqrt{ } 2, \theta=0, \phi=\pi / 4\right\} ;
\end{aligned}
$$




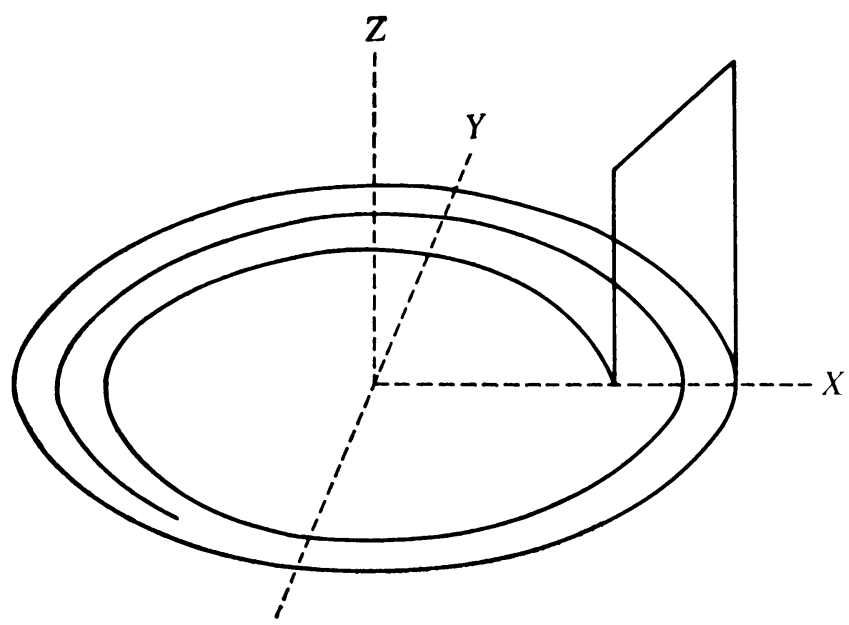

FIGURE 3

It is clear that the compact continuum $C$ is arc-wise connected.

To show that $C$ is not a continuous image of the pseudo-arc, we first choose a sequence of reference points $a_{0}, a_{1}, a_{2}, \cdots$ of the ray $R$, where $a_{0}$ is the point whose spherical co-ordinates are $\left(2-2^{-4}, 0, \pi / 2\right)$ and is referred to as the first point of $R$, and $a_{0}, a_{1}, a_{2}, \cdots$ are the successive points of intersection of $R$ with the set consisting of the union of the $x$ and $y$ axes.

Now, suppose that the continuum $C$ is a continuous image of the pseudo-arc and, noting Theorem 3.4 , let $P=\left(p_{1}, p_{2}, \cdots, p_{n}\right)$ and $Q=\left(q_{1}, q_{2}, \cdots, q_{m}\right)$ be two $p$-chains of a sequence associated with $C$ such that the first link of each $p$-chain of the sequence contains the point $a_{0}$. In particular, we choose the $p$-chains $P$ and $Q$ such that each link of $P$ has diameter less than $\varepsilon=2^{-2 m}$, each link of $Q$ has diameter less than $1 / 4$ and $P$ is a normal refinement of $Q$. Then, from this choice of restriction of the diameters of the links of $P$, there is either (1) a sub- $p$ chain $P(h, k)$ of $P$ such that $p_{h}$ has arc-distance less than $2 \varepsilon$ from $a_{0}, p_{k}$ has arcdistance less than $2 \varepsilon$ from $a_{m}$ and each link of $P(h, k)$ lies in $\left[a_{0}, a_{m}\right]$ and has arc-diameter less than $2 \varepsilon$, or (2) a similar statement can be made in which $a_{0}$ is replaced by $a_{2 m}$. In either case we may choose a $2 \varepsilon$-sequence of points $c_{h}, c_{h+1}, \cdots, c_{k}$ such that each point $c_{i}$ of this sequence is an element of the link $p_{i}$ of $P$ and the arc-diameter of the set of points of this sequence is greater than $m$. But this situation is similar to the one considered in the discussion of $K$ in the previous section, so that we may choose a corresponding sequence of points from the links of $Q$ and proceed using the same method of proof as that used in Theorem 5.2(c). Therefore, we conclude that $C$ is not a continuous image of the pseudo-arc.

EXAMPLE 6.3. A compact metric continuum $D$ which is semi-locally connected and aposyndetic, but which is not a continuous image of the pseudo-arc.

Definition of $D$. In terms of cylindrical co-ordinates the continuum $D$ is defined to be the closure of the set $\left\{(r, \theta, z): r=2-2^{-\theta / 2 \pi}, \theta \geqq 4 \pi, 0 \leqq z \leqq 1\right\}$. 


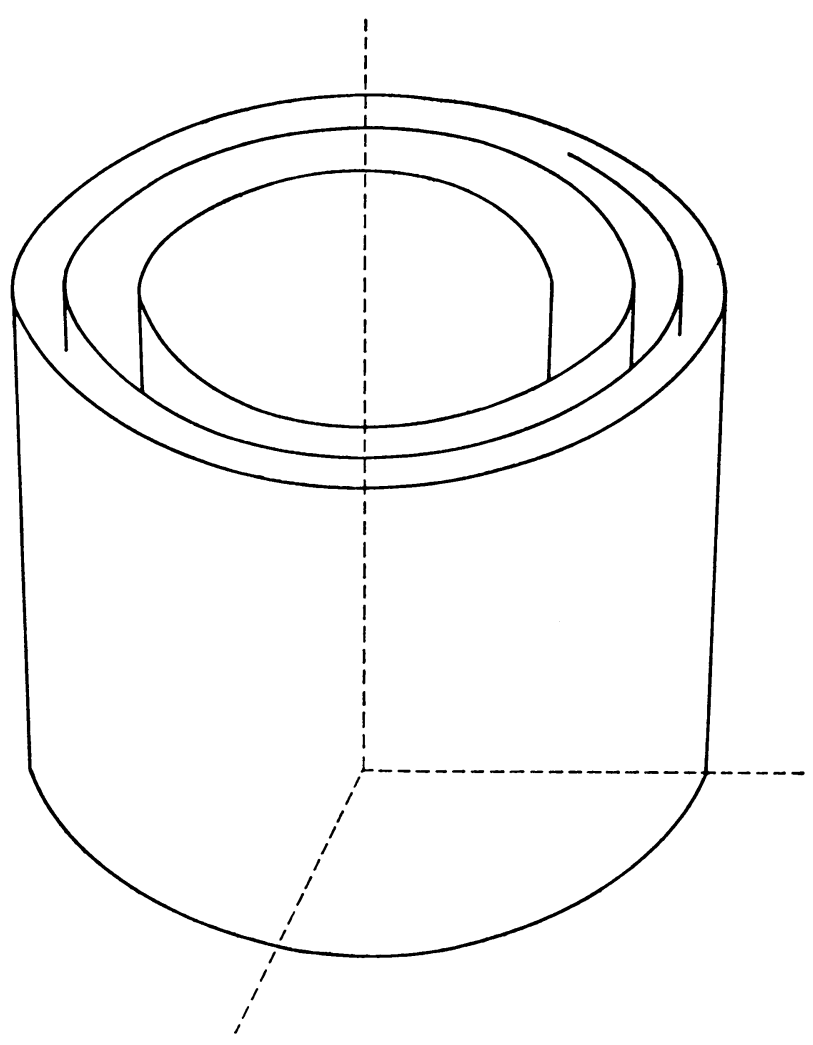

FIGURE 4

It is easily seen that the compact continuum $D$ is semi-locally connected and can be shown that the projection of this continuum onto the $(z=0)$-plane fails to be a continuous image of the pseudo-arc. Therefore, since the projection mapping is continuous, $D$ is not a continuous image of the pseudo-arc.

\section{BIBLIOGRAPHY}

1. R. H. Bing, A homogeneous indecomposable plane continuum, Duke Math. J. 15 (1948), 729-742.

2. Concerning hereditarily indecomposable continua, Pacific J. Math. 1 (1951), 43-51.

3. - The pseudo-arc, Summary of Lectures and Seminars, Summer Institute on Set Theoretic Topology, Madison, Wis., 1955, Amer. Math. Soc., Providence, R. I., 70-73.

4. M. K. Fort, Jr., Images of plane continua, Amer. J. Math. 81 (1959), 541-546.

5. H. Hahn, Mengentheoretische Characterisierung der Stetige Kurven, Sitzungsberichte Akademie der Wissenschaften Vienna 123 (1914), 2433.

6. F. B. Jones, Concerning aposyndetic and non-aposyndetic continua, Bull. Amer. Math. Soc. 58 (1942), 137-151.

7. B. Knaster, Un continu dont tout sous-continu est indécomposables, Fund. Math. 3 (1922), 247-286. 
8. S. Mazurkiewicz, Sur les lignes de Jordan, Fund. Math. 1 (1920), 166-209.

9. J. Mioduszewski, Functional conception of snake-like continua, Fund. Math. 51 (1962), 179-189.

10. E. E. Moise, An indecomposable plane continuum which is homeomorphic to each of its nondegenerate subcontinua, Trans. Amer. Math. Soc. 63 (1948), 581-594.

11. Z. Waraszkiewicz, Sur quelques invariants des transformations continues, Fund. Math. 23 (1934), 172-189.

12. G. T. Whyburn, Analytic topology, Amer. Math. Soc. Colloq. Publ. Vol. 28, Amer. Math. Soc., Providence, R.I., 1942.

BRIGHAM YOUNG UNIVERSITY, Provo, Utah 